\title{
OBSERVATORIO
}

\section{E-GOBIERNO: ¿MODERNIZACIÓN O FOSILIZACIÓN DE LA ADMINISTRACIÓN?}

\section{Adela d'Alòs-Moner}

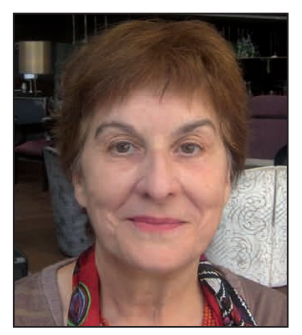

Adela d'Alòs-Moner, socia fundadora de Doc6, tiene una larga trayectoria tanto en centros públicos como privados. Ha sido directora de la biblioteca de ciencias de la Universitat Autònoma de Barcelona y coordinadora de distintos proyectos europeos. Ha impartido clases en diversas universidades y ha sido presidenta del Col-legi Oficial de Bibliotecaris-Documentalistes de Catalunya (Cobdc).

Doc6, Consultores en recursos de información http://www.doc6.es aalos@doc6.es

\section{Resumen}

Después de una introducción global sobre conceptos como egobierno, edemocracia o egobernanza, se hace una breve reflexión sobre la situación de la administración pública española en relación con internet. Se plantea la oportunidad que representan las tecnologías de la información y la comunicación para llevar a cabo reformas en profundidad en los procedimientos administrativos. De no hacerlo, se corre el riesgo de "fosilizar" la administración y realizar únicamente cambios superficiales que no contribuyen a la necesaria mejora de la eficiencia y del servicio a empresas y ciudadanos.

\section{Palabras clave}

Administración pública, Gobierno electrónico, Transparencia administrativa, Tramitación online, E-gobernanza, E-gobierno, E-democracia, E-administración.

\section{Title: E-government: modernization or fossilization of the administration?}

\begin{abstract}
After a general introduction to concepts such as eGovernment, eDemocracy and eGovernance, a brief reflection on the situation of the Spanish public administration in relation to the internet is offered. The opportunities that information and communication technologies offer to carry out deep reforms in administrative procedures is discussed. If we do not seize these opportunities we risk "fossilizing" the administration, making only superficial changes that do not make the needed improvements in efficiency and the services to businesses and citizens.
\end{abstract}

\section{Keywords}

Public administration, Electronic government, Administrative transparency, Online transactions, E-governance, E-Government, E-democracy.

d’Alòs-Moner, Adela. “E-gobierno: ¿modernización o fosilización de la administración?”. El profesional de la información, 2011, mayo-junio, v. 20, n. 3, pp. 245-249.

\section{E-gobernanza, e-gobierno, e-democracia}

En esta editorial se quiere reflejar el estado de la administración pública española en internet. Estamos en una encrucijada que puede derivar en un fortalecimiento de la modernización y democratización de la administración pública o en un anquilosamiento barnizado de modernidad.

En los últimos años se han consolidado en la administración pública las expresiones e-gobierno (eGovernment en inglés), e-democracia (eDemocracy), eProcurement y, como

Artículo recibido el 03-04-11

Aceptación definitiva: 14-04-11 concepto más amplio que los engloba a todos, e-gobernanza (eGovernance).

El e-gobierno es ofrecer a través de internet información y servicios. La e-democracia engloba las formas de comunicación entre electos y elegidos y se asocia con votaciones y referendums online. El eProcurement se asocia a la gestión de trámites a través de internet, especialmente compras y ventas.

Rogers W.O Okot-Uma, consultor del Commonwealth Secretariat, en el artículo Electronic governance: re-inventing 
good governance ${ }^{1}$, remarca la oportunidad que ofrece la egobernanza a los gobiernos para reinventarse, para aproximarse al ciudadano y para crear alianzas y redes con distintas comunidades de intereses y experiencias.

La entiende como una iniciativa que debe moverse a distintos ámbitos - local, estatal- que debe explotar las posibilidades de las tecnologías de la información y las comunicaciones para conseguir la buena gobernanza.

En relación con estos conceptos y para intercambiar experiencias entre distintas administraciones, han aparecido muchos grupos y forums, gran parte de ellos de países anglosajones.

A título de ejemplo podemos citar:

\section{Center for Governmental Studies}

http://www.cgs.org

Organización sin fines de lucro, no partidista, fundada en 1983 , busca crear soluciones innovadoras en medios políticos y ayudar a los individuos participar más eficazmente en sus comunidades y los gobiernos.

\section{DoWire, Democracies online}

http://dowire.org

Blog y lista de distribución puestos en marcha en enero de 1998 para estar al día de iniciativas que se realizan en todo el mundo sobre democracia online.

\section{E-Democracy.org}

http://www.e-democracy.org

Fundada en 1994, mantiene forums y blogs

\section{PublicUs.net}

http://www.publicus.net/articles/edemresources.html

Lista de recursos muy completa del consultor Steven Clift, relacionado también con DoWire y E-Democracy.

Por último, la Comisión Europea mantiene una web con 40 foros temáticos, blog, directorio de expertos, casos prácticos, eventos, etc.:

http://www.epractice.eu

\section{¿Podemos realmente hablar de e-gobierno o de e-democracia?}

Se ha escrito mucho y cada día aparecen nuevos informes y artículos sobre e-gobierno y cada vez más se manifiesta la necesidad de "reinventar" la manera de gobernar. Se constata que las tecnologías de la información y las comunicaciones deben ser una oportunidad de cambio en la administración pública que les permita:

- mejorar los procesos internos;

- mejorar los servicios;

- intensificar la interacción con los ciudadanos y las empresas;

- potenciar el desarrollo económico.

La inversión en infraestructura que muchos gobiernos están realizando responde a una visión basada en que el crecimiento económico, el cual sólo será posible si se dispone de un acceso a la Red rápido y seguro.

En la web de la Comisión Europea se puede leer:
"La administración electrónica se basa en el uso de las herramientas y sistemas de información y comunicación (TIC) para proporcionar mejores servicios públicos a ciudadanos y empresas. Las TIC ya son muy utilizadas por los órganos de gobierno, así como por las empresas, pero la administración electrónica implica mucho más que las herramientas. También implica repensar las organizaciones y procesos, así como cambiar el comportamiento de modo que los servicios públicos se presten de manera más eficiente a las personas que los necesitan. Bien implementada, la administración electrónica permite a todos los ciudadanos, empresas y organizaciones llevar a cabo sus tratos con el gobierno con más facilidad, más rápidamente y a menor costo".

http://ec.europa.eu/information_society/activities/egover nment/index_en.htm

Nadie duda del importante papel que las TIC pueden tener en las administraciones públicas y en su relación con los ciudadanos y las empresas. No está tan claro que se sea tan consciente que las TIC pueden representar una oportunidad de cambio, para "reinventar" la manera de gobernar.

Si analizamos la tramitación online, sin duda uno de los servicios que las administraciones deben asegurar y donde muchas de ellas están aún hoy centradas, vemos que es necesario tener una visión amplia que asegure que se aprovecha la puesta en funcionamiento de un trámite por internet para realizar una reingeniería del proceso en su globalidad. Por ejemplo, ¿qué datos se preguntan? ¿dispone ya la administración de estos datos?, ¿̇obliga el marco legal a pedirlos? ¿si no es necesario pedirlos, son datos que después se tratan y se elaboran? Al formularse muchas de estas preguntas se comprueba fácilmente que muchos trámites se realizan usando viejos modelos, sin pensar cómo pueden mejorarse, simplificarse o explotarse para la optimización global del servicio.

\section{Evolución de la administración pública en internet}

Desde mediados de 1990, cuando empiezan las primeras webs en las administraciones públicas, se ha pasado por 4 fases que se muestran en la figura 1.

Una vez cubierta una primera fase consistente en facilitar información sobre la propia administración, los ejes de actuación se han centrado en potenciar la tramitación online. En un primer momento, con limitadas posibilidades de interacción (por ejemplo, debe imprimirse el formulario para después presentarlo presencialmente), y posteriormente con la posibilidad de realizar íntegramente el trámite por internet. Finalmente las webs de las administraciones se plantean también como un instrumento de participación ciudadana: forums, posibilidad de dejar mensajes en la red, de enviar mensajes electrónicos a cargos políticos, de intervenir en

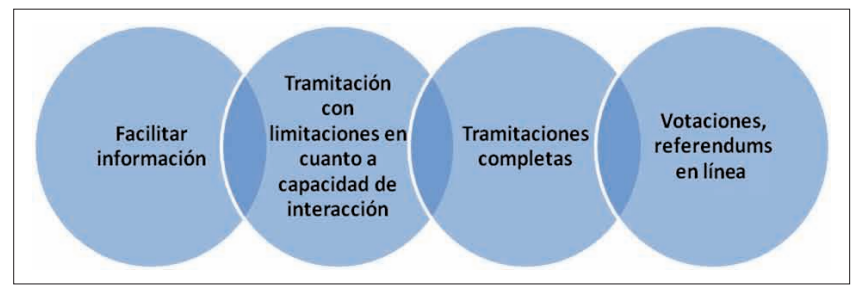

Figura 1 


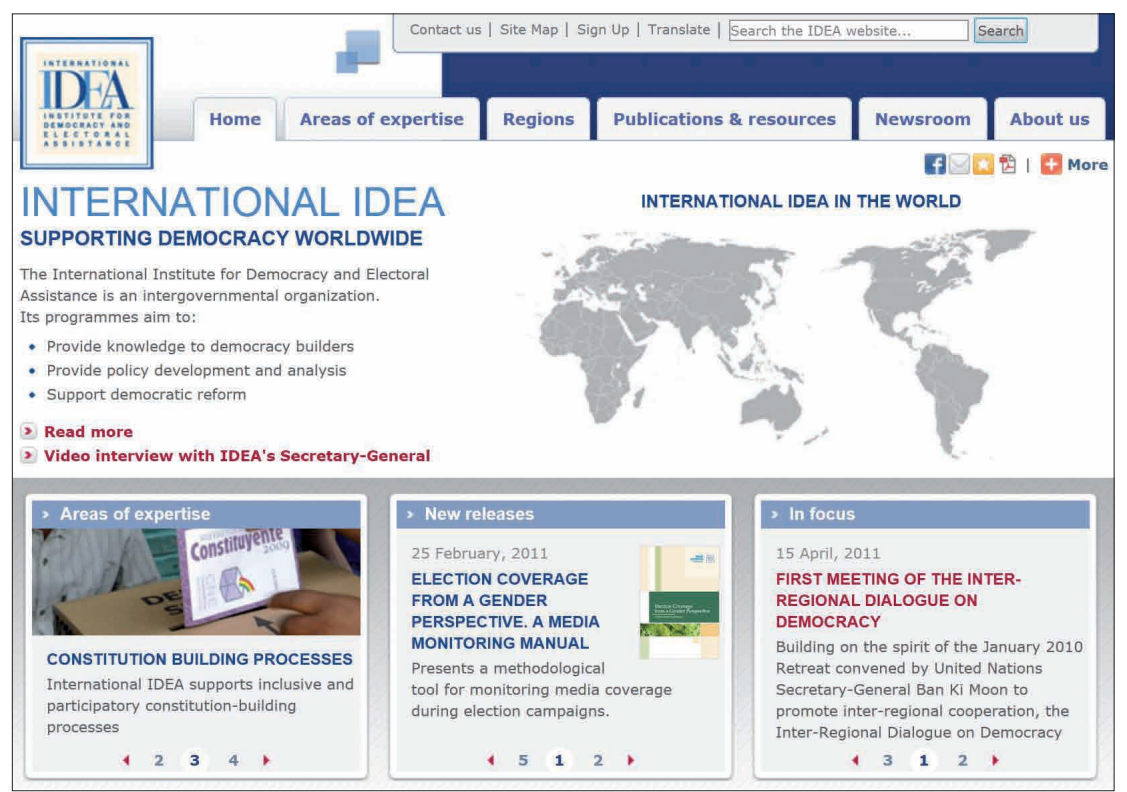

Sobre este tema es necesario mencionar un artículo publicado en el año 2000, Top ten e-democracy "to do list" for governments around the world del citado Steven Clift.

http://www.publicus.net/articles/egovten.html

El artículo señala que los gobiernos tienen ahora la posibilidad de romper la mentalidad basada en "nosotros" y "ellos" y apostar por una administración como una institución del pueblo. Según Clift los diez aspectos que deben llevarse a cabo para poder hablar de e-democracia son:

1. Informar de las reuniones públicas a través de internet de manera sistemática y efectiva.

los plenos municipales a través de la Red, etc. Donde se están realizando avances, gracias a las normativas sobre privacidad de datos y firma digital, es en la posibilidad de votar online. De momento son pocas las experiencias, pero ya se vislumbra como una vía de futuro cercano.

Una de las primeras iniciativas se remonta al año 1994: Minnesota E-Democracy. Se trata de una organización que tiene como misión mejorar la participación democrática de los ciudadanos de Minnesota a través de las redes de información y las tecnologías de las comunicaciones.

http://forums.e-democracy.org

Entre las organizaciones de referencia podemos citar el intergubernamental Instituto Internacional para la Democracia y la Asistencia Electoral (IDEA), que tiene su sede en Estocolmo. http://www.idea.int

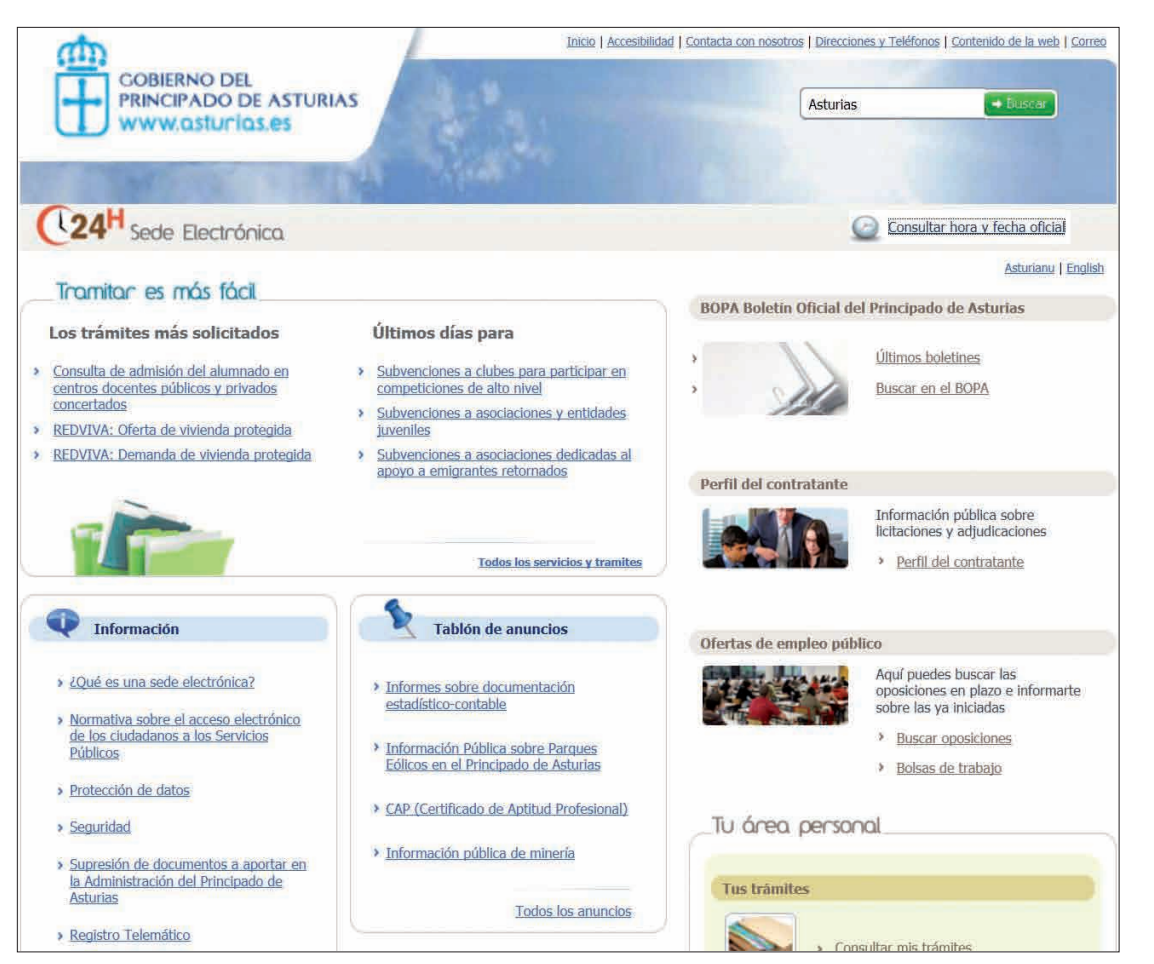

Sede electrónica del Principado de Asturias, una de las mejores de España, https://sede.asturias.es
2. Introducir el "botón democrático" en la primea página de la web corporativa que explique la misión y los objetivos del ente, las personas de dirección que toman las decisiones, las principales leyes que afectan a los ciudadanos, etc.

3. Implementar el "servicio democrático" que facilite trámitesonline, realiceencuestasatravés dela Red, recojaopiniones deciudadanos, potencie su interacción con la administración, etc.

4. Acabar con el "déficit de la democracia online"; es decir, invertir en tecnología y en infraestructura de comunicaciones.

5. Potenciar los niveles de representación a través de internet creando "despachos virtuales" que permitan recoger e intercambiar opiniones de ciudadanos.
6. Impulsar las posibilidades de canal bidireccional de internet y contestar de manera rápida y efectiva a los correos electrónicos. Definir una política clara al respecto.

7. Realizar consultas a través de la red que sirvan para educar a los ciudadanos a participar más en "el bien público".

8. Desarrollar normativas y leyes que regulen la e-democracia.

9. Educar a los cargos electos en el uso de internet en su trabajo diario. En este sentido el mismo autor sugiere que debe asegurase que los cargos electos han leído su artículo "Top ten tips for wired elected oficials" http://www.publicus.net/articles/weos. html

10. Crear aplicaciones para asegurar un único punto de acceso a la Administración. Compartir con otras administraciones de todo el mundo. 


\section{Papel de la administración pública}

Estamos en una nueva realidad en la que la administración pública tiene un papel múltiple:

- proporcionar el acceso y asegurar la transparencia administrativa facilitando la relación entre ciudadanos y empresas con la propia administración. Mientras que la administración tiene la obligación de dar a conocer información de interés público, el ciudadano tiene el derecho a acceder a determinadas informaciones de la administración que le atañen directa o indirectamente;

- tener un papel de liderazgo, democratizando el acceso a la información. Para ello debe facilitar acceso y formación a los ciudadanos y asociaciones a los sistemas de información y potenciar el uso extensivo e intensivo de las TIC en las empresas, especialmente las pymes.

- crear las infraestructuras necesarias para la sociedad de la información y evitar el gap o brecha digital y facilitar la participación en la sociedad de la información.

- asegurar la privacidad de los datos y un acceso fiable y seguro a la información y a los trámites.

- potenciar el sentido de comunidad y la identidad cultural a través de las TIC.

\section{Transparencia administrativa}

La administración dispone de mucha información que es de interés general o de interés específico para personas o empresas. Hoy tiene en internet un importante instrumento que le permite ponerla al alcance de todos o de quien la precise.

Aunque ya hace años que se ha planteado el derecho a la información pública -cabe recordar, por ejemplo, el informe realizado por la Unesco en 2004 "El derecho de los ciudadanos a la información pública ${ }^{2 \prime}$ - de momento, ni la Comisión Europea ni el Estado español ha legislado estos aspectos de manera clara.

Desde que en diciembre de 2009 Obama lanzó la iniciativa "About open government", este tema ha empezado a adquirir mayor relevancia.

http://www.whitehouse.gov/open

\section{Situación de España}

Sin duda constatamos que durante los últimos años ha habido un avance importante en los servicios que las distintas administraciones ofrecen a los ciudadanos y empresas.

Han pasado poco más de 15 años desde que la mayoría de las administraciones públicas empezaron a tener sus webs online $y$, durante este período ha habido bastantes progresos.

En España destacan el Gobierno de Asturias que tiene una de las webs mejor valoradas, la Generalitat de Catalunya o los Ayuntamientos de Zaragoza, Alcobendas, Barcelona o Irún, por citar algunos de los más significativos.

http://www.asturias.es

http://www.gencat.cat

http://www.zaragoza.es

http://portal.alcobendas.org/es

http://www.bcn.cat

http://www.irun.org

El informe United Nations e-government survey que periódicamente ofrece Naciones Unidas -el último fue publicado en 2010- revela que España se encuentra en una buena posición. En e-government se sitúa en la posición número 9 del ranking global, por delante de Singapur, Francia o Suecia. Y en e-participación está en tercer lugar, superada sólo por la Republica de Corea y Australia. Por lo que se refiere a Europa, España se encuentra en quinto lugar, después del Reino Unido, Países Bajos, Noruega y Dinamarca.

http://www.unpan.org/egovkb/global_reports/08report. htm

Estos resultados pueden sorprender. Responden en gran medida a la alta valoración que hace el informe del portal www.red.es. En junio de 2010 la Secretaria de Estado para la Función Pública, Consuelo Rumí, recogió en Barcelona el premio de Naciones Unidas al Servicio Público en reconocimiento a la mejora de calidad de los servicios que la Administración General del Estado presta a los ciudadanos a través de internet. Los ejes que destacó fueron:

- Ley 11/2007 de acceso electrónico de los ciudadanos a los servicios públicos;

- promoción del DNI electrónico;

- creación de infraestructuras y de servicios;

- campañas de información hacia el ciudadano y de formación hacia el empleado público;

- avances en la colaboración, coordinación y cooperación entre los diferentes niveles de la Administración.

\section{Objetivos de futuro}

Debemos evitar caer en triunfalismos: quedan bastantes aspectos que nos indican que aún queda mucho camino por recorrer.

En primer lugar, la tramitación online: es cuanto menos chocante que la mayoría de las administraciones en España se han fo- 
calizado sobre todo en los trámites online más complejos de llevar a cabo y que, en concreto, son los que generan ingresos. Por el contrario, los relacionados con subvenciones o registros, más fáciles de elaborar, son los menos desarrollados. Es evidente que la administración requiere de los impuestos de los ciudadanos y empresas pero ello no debería comportar que trámites fáciles de realizar y de gran importancia para la economía -por ejemplo, el registro de actividades empresariales-, no esté disponible en muchas comunidades. En cambio, un $88 \%$ ofrecen el trámite de "tasas autoliquidables". Así lo evidencia un estudio publicado por la Fundación Orange: http://fundacionorange.es/areas/25_publicaciones/eadministracion2009.pdf

Algunos trámites municipales que muchos ciudadanos agradecerían y que son fáciles de implementar -por ejemplo, reserva de plazas para escuelas o guarderías- no se encuentran disponibles en muchos casos.

Quedan también por resolver aspectos relacionados con la interoperatividad de manera que una parte de la administración no reclame al ciudadano datos de los que ya dispone otra. La voluntad de servicio público debe ganar terreno. Es necesario que las administraciones compartan recursos y se avance realmente hacia la "ventanilla única" que facilite al ciudadano un único punto de acceso. Las administraciones deben avanzar en el cruce de información para evitar que ciudadanos y empresas deban ir de una a otra (aunque sea virtualmente) para resolver temas de los que la administración tiene ya la información.

También hay que destacar la falta de transparencia. Si bien se ha empezado a dar algún paso adelante para facilitar información sobre sus propias actuaciones y sobre datos generales de los que dispone y puede parecer que se tiene información, esto no siempre es así.

El profesor del IESE Eduardo Martínez-Abascal en su blog ${ }^{3}$ plantea preguntas como:

“¿Cuánto gasta la Administración Pública en España? Difícil de saber. Busque en Google, pregunte por ahí. Como mucho, obtendrá el dato de los presupuestos generales del Estado (unos 350 millardos). Pero éstos no cubren parte de los gastos de las comunidades autónomas ni de los ayuntamientos, que en un estado descentralizado suponen buena parte del gasto público. Doy el dato: el gasto de la administración pública en el 2009 fue de 483 millardos. Las empresas son analizadas a partir de sus resultados, es decir de su cuenta de resultados y balance.

Pregunta: ¿ha visto usted alguna vez la cuenta de resultados del Estado Español? ¿Ha oído discutir en el Parlamento sobre el cierre del ejercicio presupuestario? Nos cansamos de discutir el presupuesto, pero nunca rendimos cuentas sobre lo que ha pasado con el presupuesto. Sólo en los últimos meses, y ante la preocupación europea por el déficit público español, empezamos a dar algunos datos parciales sobre el cierre del ejercicio. Propuesta: que cada año se presenten las cuentas del ejercicio anterior y se discutan en el Parlamento".

Hay aún grandes distancias entre España y países como Suecia y Finlandia donde la concepción de la transparencia está enraizada desde hace años. En Finlandia, el "Act on the openness of government activities", que regula el derecho del ciudadano a recibir información de la administración pública, data de diciembre de 1999; refuerza normativas anteriores y obliga con más rigor a las autoridades públicas. Según esta norma "los documentos públicos deben ser públicos". De no ser así, los documentos que genera el gobierno y se considere que no deben ser públicos, deberán estar regulados por ley.

Pero más allá, esta norma incluye la necesidad por parte de la administración de seguir "mejores prácticas" relacionadas con la información y con la gestión de los documentos: revisión y calidad de documentos públicos y protección y seguridad de los datos.

\section{Los profesionales de la información}

En este contexto los profesionales de la información podemos y debemos jugar un papel importante: contribuyendo a potenciar el uso de metadatos que permitan la interoperabilidad en cuanto a la búsqueda cruzada de contenidos, apoyando la adecuación a la Ley 11/2007 a través de una correcta gestión de la documentación, con la creación de taxonomías y vocabularios controlados como, por ejemplo, el del Reino Unido "Integrated public sector vocabulary" (IPSV). http://www.esd.org.uk/standards/ipsv_internalvocabulary

Por otro lado, desde las bibliotecas públicas se puede hacer una gran tarea ayudando a la gente a que realice sus trámites con la e-administración.

Pero estos temas serían objeto de otro editorial.

\section{Notas}

1. Commonwealth Secretariat London.

http://webworld.unesco.org/publications/it/egov/word bank\%20okot-uma.pdf

2. http://www.unesco.org.uy/ci/fileadmin/comunicacioninformacion/derechoinformacion.pdf

3. http://blog.iese.edu/martinezabascal/2011/02/16/cuen tas-publicas-mas-transparencia 


\section{doc6 en 30 segundos}

\section{Nuestra misión}

Aportar soluciones para mejorar la eficiencia de empresas y organizaciones a través de una eficaz gestión de la información y del conocimiento.

\section{La empresa}

Creada en 1988. Le aportamos más de $\mathbf{2 0}$ años de experiencia.

a Consultoría en gestión documental, de la información y del conocimiento

- Sistemas de Gestión Documental: mapas documentales, cuadros de clasificación de archivos, concreción de funcionales, etc.

- Intranets, Extranets. Arquitectura y usabilidad web. Taxonomías

- Servicios profesionales adecuados a cada necesidad

- Soluciones en gestión documental

- Recursos electrónicos de información. Representante en España de OCLC

a Formación personalizada

\section{Los 6 valores de doc6}

Los valores por los que se rige la actividad de doc6 definen nuestro código ético, nuestros métodos de trabajo y organización, la cultura de la empresa. Son los siguientes:

- Excelencia en calidad

- Innovación permanente. Creatividad

a Cooperación y trabajo en equipo

Confidencialidad

- Profesionalidad y honestidad con clientes y proveedores

Responsabilidad social

\section{Los clientes}

El principal activo de la empresa, junto con sus empleados.

Más de 1000 clientes: empresas, laboratorios, administraciones públicas, universidades, hospitales, bufetes de abogados, museos, fundaciones, colegios profesionales.

\section{Nuestros profesionales}

Un equipo de más de $\mathbf{1 0 0}$ profesionales.

Una eficaz suma de conocimientos: informáticos y documentalistas con experiencia.

El $90 \%$ son licenciados superiores o diplomados en informática o documentación. 